7. The pains in the groin and thigh much diminished, but increased down the inner part of the leg, in the course of the saphenus nerve, returning every few minutes with great severity; vision affected; pupils slightly dilated; bowels open. Continues six grains of the extract of belladonna during the day and night.

8. Very bad night from the pains along the leg when warm in bed being increased; neuralgic pains continue abated. Influence of belladonna over the irides slight. Ordered to apply a blister along the leg in course of saphenus nerve; the cuticle to be raised, and the abraded surface dressed with the stronger belladonna ointment three times a-day.

10. The blister has risen well; the application of the belladonna ointment has produced an almost unbearable degree of "tingling" and formication; pupils much more dilated; slight headach ; bowels very open. Ordered to keep the blistered surface discharging by the application of the savine ointment and to continue the application of the belladonna ointment twice a-day, taking the same number of pills. This ointment was persevered in until the $17 \mathrm{th}$, the medicated ointment producing the same degree of "tingling," accompanied with a considerable degree of diminished natural sensibility; the nervous pains much diminished. Is anxious to have the blistered surface healed. To apply simple spermaceti ointment, omitting every thing else.

19. Blistered surface healing; neuralgic pains and those along the leg "almost entirely gone;" has very slight pain in the back part of the hip; general health improved; rest still disturbed on moving the leg during sleep; is on the whole much improved.

23. Is quite free from all pain; blistered surface healed. Ordered to apply on the leg a belladonna plaster.

25. Rests well; is quite free from all neuralgic pain; general health improving; walks readily with a stick, but has a slight halt in consequence of the limb being still very weak and wasted.

In the preceding case the doses of the extract of belladonna amounted, for a considerable number of days, to three grains in the twenty-four hours, and as the patient himself believed, produced slight purging; but this having occurred during the recent hot weather, it is doubtful if it was produced by that medicine, or from the disposition which at that time existed here to diarrhcea. The belladonna has been observed occasionally to act upon the bowels. In either case it is probable that the apparent insusceptibility of this patient to the usual effects of the medicine may be attributed to the slight diarrhoea accompanying its administration. It was curious to observe that the internal administration of the belladonna influenced much more favourably the neuralgia of the anterior crural nerve and its branches upon the thigh than that of the saphenus nerve, and that the neuralgia affecting this nerve was speedily relieved by the local application of the remedy. So that it appears desirable that the local application in obstinate cases should be, when it is possible, combined with the internal administration of this remedy.

In the preceding papers it has been attempted to elucidate the beneficial effects of the belladonna upon the morbid affections of the nerve of sensibility; in the succeeding will be pursued the same investigation touching its influence upon the disordered action of the motor nerves, such as produces chorea and tetanus.

Nottingham, Sept. 27, 1843.

\section{ABORTION. \\ THE PLACENTA RETAINED SIX WEEKS.}

To the Editor.-Sir: On the 21st of July last I attended Mrs. T. during a miscarriage. The foetus was extruded with very little pain, and the mother scarcely lost a drop of blood. The fortus appeared to be at about the fifth month of development, and the funis was somewhat thicker than a goose-quill. On passing my finger along the cord I traced it into the uterus, without being able to reach the placenta. Pressure was made on the abdomen, and, upon making slight traction, the cord tore away. As the state of the os uteri would not admit of the introduction of two fingers for the purpose of extracting the placenta, I had recourse to the ergot of rye, which was given, in repeated doses, without any obvious effect. During the succeeding ten or twelve days $i$ watched the patient carefully. She recovered without any untoward symptom. The os uteri closed, and a slight lochial discharge, without fetor, continued during two or three days.

On the first of September, being exactly six weeks after the birth of the foetus, I received a hurried message from Mrs. T. I found her lying on her back, throwing her arms wildly about the bed, and uttering a low moaning noise. Profuse hæmorrbage was going on, although the os uteri was very small, and no presentation could be felt. The hæmorrhage was arrested by cold wet cloths applied to the abdomen and vulva, and the patient soon rallied under the use of brandy and water. She had several attacks of sickness and approaching syncope during the night, but remained free from pain or hæmorrhage, until five o'clock next morning, when a severe flooding again occurred, accompanied with expulsive paios. The os uteri was now dilated to the size of a shilling, and a fleshy mass presented, having the feel of a placenta, but less distinctly lobulated, and at one part resembling the 
tense membranes of an ovum. As soon as females as to the danger they incur when two fingers could be introduced within the trusting their lives, at such a critical period, uterus a fibrous substance, perfectly smooth to this too commonly employed, but geneat one part, was extracted. I have no rally careless, class of persons. I am, Sir, doubt that this was the placenta, which Your obedient servant, ought to have been thrown off six weeks before, although, from the cessation of its function, its appearance was considerably changed.

The points worthy of notice in this case are, first, the length of time during which the placenta was retained in the uterus without giving rise to any severe symptoms; and, secondly, when it presented at the os uteri, the difficulty of deciding whether there might not be an ovum above the placenta, either from there having been twins in the first instance, or from a subsequent impreguation. However, the indication was to empty the uterus, and when the fingers were introduced for that purpose, the nature of the case was evident. The placenta was adherent to the posterior part of the uterus. Yours, faithfully,

South Shields, Oct. 4, 1843.

W. EMMERson.

\section{HANDY-WORK OF A MIDWIFE.}

To the Editor.-Sir : Allow me, through the medium of THE LANCET, to draw attention to the following unusual case, as showing the hazard to which a lying-in woman may be exposed from the violence of an ignorant aud incompetent midwife. I was lately summoned to Maria Clarke, a poor person residing at Selston, a few miles distant, who was reported to have miscarried, and to be then in a very dangerous situation. I was not a little surprised, on my arrival, at having presented to me the headless body of a fine fresh infant, of about the fifth month of gestation, with the funis torn off close to the umbilicus, the head and placenta remaining in the uterus. After several inquiries I ascertained that a midwife from the neighbourhood had officiated, but the parties present were evidently unwilling to say much about the occurrence. On examination, the os uteri was found firmly closed, and no trace of the ruptured cord could be detected. There was no hæmorrhage, all pains had completely subsided, and the woman was extremely weak. After the exhibition of a stimulant the os uteri was gradually dilated, and the escape of the head soon after effected. The placenta occasioned some difficuliy in its removal, from being partially adherent. The nterus contracted slowly after the withdrawal of the hand, and no flooding followed. The restoration to health was very protracted. The foetus is in my possession; the atlas remains attached to the head, and the dentata to the other cervical vertebræ, exactly as the laceration had occurred. A case like the above should afford a salutary caution to
Samuel Williams, M.R.C.S. Eastwood, near Nottingham.

Sept. 14, 1843.

\section{FATAL CASE OF CANCRUM ORIS,} IN WHICH

\section{AN INQUEST WAS HELD ON THE BODY.}

To the Editor of THE LANCET.

$\mathrm{SIR}_{\mathrm{R}},-\mathrm{S}$. Ridly, aged two years and three months, was brought to my house, by her mother, on Saturday, the 16th of September. The child had an expression of heaviness about the eyes, the skin was hot, and the pulse quiet. The mucous membrane of the mouth was in an unhealthy state, and the gums were spongy. There were blotches upon the body, resembling the pustular form of scabies. The child was of a cachectic habit, arising from the combined influence of an unhealthy locality (Windsor-court, in the Strand), and defective nutrition. It was scarcely possible, indeed, for it to have been otherwise from the terms upon which the parents of the child live. I have often pitied their poor children. Within the last few months the mother nearly lost her life from flooding, in consequence of a miscarriage, occasioned, as she stated to me, " by the kicks and blows and brutal treatment of her husband," and the external marks upon her body gave ample proofs of acts of violence. I have been called upon to furnish a certifi. cate of her inability to appear at Bow-street police-office, on account of family differences. I think it necessary to state these facts; they are important, in a medico-legal point of view, for forming a just opinion on the con. ficting testimony in the evidence on the case, but still more in reference to the effects of imperfect nutrition, and the formation of the cachectic habit. How the children, living in such a vitiated atmosphere, and neglected as $I$ have seen them, could escape constitutional deterioration, it is difficult to conceive.

I prescribed for the child a mixture of magnesia and soda, three alterative pow. ders, each containing four grains of powdered rhubarb, two grains of dried carbonate of soda, and two grains of mercury with chalk, every other night; and some compound sulphur ointment to be applied to the blotches. One osly of the powders was given. On the Monday morning I was asked to visit the child in Windsor-court; $I$ found the eruption of measles was coming out, but not freely. The child was in a low, drowsy state; pulse quick, but weak ; skin hot and dry; the bowels much relaxed, with pneu. 